\title{
Correction of nasolabial fold by combination of polydioxanone thread and hyaluronic acid filler: a case report
}

\author{
Kyoungjin Kang, MD, $\mathrm{PhD}^{1} \mathbb{1}$, Hanwool Choung, DDS, $\mathrm{PhD}^{2} \mathbb{1}$, Uekyoung Hwang, MD, $\mathrm{MPh}^{3} \mathbb{1}$ \\ ${ }^{1}$ Department of Plastic Surgery, Seoul Face 21 Dental Hospital, Seoul, Rep. of Korea \\ ${ }^{2}$ Department of Maxillofacial Surgery, Seoul Face 21 Dental Hospital, Seoul, Rep. of Korea \\ ${ }^{3}$ Yeline Esthetic Clinic, Seongnam, Rep. of Korea
}

\begin{abstract}
Deep nasolabial folds (NLFs) are a common concern among aging patients. Hyaluronic acid (HA) filler is the first treatment choice. However, migration, spread to the surrounding space, and unnatural contours with lateral bulges occur frequently. Polydioxanone (PDO) thread has also been used by insertion of cogged PDO threads in the midface and the fold. Unfortunately, lifting lasts for only a few weeks. In the present case, a combination procedure using HA filler and PDO thread in the shape of the mesh tube was performed to improve the deep NLF in a 67-year-old woman. The intention of this combination was to unfold the NLFs and restore their volume without lateral migration of the injected filler. NLFs were divided into three parts: the lateral folded part (LFP), medial depressed part (MDP), and central crease part (CCP). Five PDO threads $(6.0 \mathrm{~cm}$ length) were sequentially inserted into the LFP, MDP, and then under the CCP of each NLF. Total $4.0 \mathrm{ml}$ of the filler was also sequentially injected into the both NLFs in a manner similar to the mesh thread insertion. They were mainly placed at the subdermal and superficial fat layer in the entire NLF and at the submuscular layer in the regions of the lateral part of the NLF, mouth corners, and submalar area. Three months after PDO thread insertion and HA filler injection, softened NLFs without filler migration, restored volume and lifted midface with a natural and charming smile, and reduction of perioral wrinkles were observed. These changes could be explained by the suppression of laughter movement accompanied by myo-modulation and increased firmness of the NLFs by the restoration of midface volume. These results suggest that the combination of PDO insertion and HA injection may be useful for correction of NLF.
\end{abstract}

Keywords: hyaluronic acid filler; mesh tube type; myo-modulation; nasolabial fold; polydioxanone thread

\section{Introduction}

Aging changes the midfacial fat compartments. Skin and superficial fat compartments' sagging and absorption of the deep fat layer and bone cause facial contour collapse, resulting in deep grooves in the face [1]. Moreover, aging leads to hyperactivity of the muscles of the face, which contracts strongly to compensate for changes in the skeleton of the face [2].

Deep nasolabial folds (NLFs) mainly appear due to the sagging of both the skin and the superficial malar fat pad following the decreased volume of deep medial cheek fat (DMCF). In addition, the contractility of the lip elevator muscles, such as the levator labii superioris alaeque nasi (LLSAN), levator labii superioris, zygomaticus minor, and zygomaticus major, play an

Received November 19, 2021; Accepted December 15, 2021

Corresponding author: Uekyoung Hwang

E-mail: vjukhome@daum.net

This is an Open Access article distributed under the terms of the Creative Commons Attribution Non-Commercial License (http://creativecommons.org/licenses/by-nc/4.0), which permits unrestricted non-commercial use, distribution, and reproduction in any medium, provided the original work is properly cited.

Copyright (C) 2021 Korean Society of Korean Cosmetic Surgery and Medicine (KSKCS \& KCCS). 
important role in the formation of deep NLFs [3]. There is also a suggestion [4] regarding whether the NLFs deepen due to the muscle fibers pulling the skin of this area (Fig. 1).

Based on our understanding of anatomical changes in NLFs, tightening the drooped skin and superficial malar fat fad and volume augmentation in the midface to compensate for the loss of DMCF have attracted attention as promising modalities for the treatment of NLFs. The most widely used modality for correcting NLFs is filler injection directly into the NLF crease [5]. Botulinum toxin injection also improves deep NLF creases by relaxing the lip elevator muscles [6].

The concept of myo-modulation has emerged to support the idea that filler placed around hyperactive muscle leads to

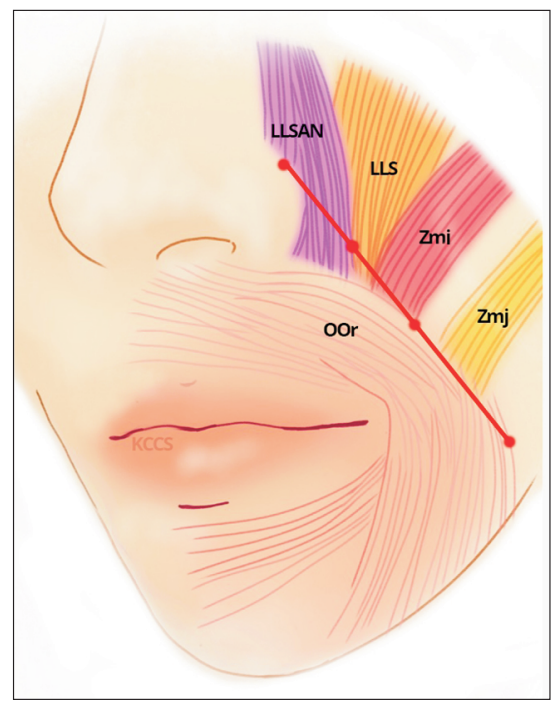

Fig. 1. Schematic position of facial muscle related to nasolabial folds. LLSAN, levator labii superioris alaeque nasi; LLS, levator labii superioris; Zmi, zygomaticus minor; Zmj; zygomaticus major; OOr, orbicularis oris muscle. Revised from the article of Hur MS, et al. (PLoS One 2020;15:e237043) [3]. stretching and relaxation of the muscles, like botulinum toxin. Dr. de Maio coined the term myo-modulation to describe the additional effect of filler injection below or above the muscle on correcting the imbalance and hyperactivity of the muscle [7]. In this regard, if the filler is injected into the deep layer of the crease of NLFs, the filler directly affects LLSAN, where the force pulling the lips upward is weakened, and the NLFs and gummy smile are improved [3].

In many cases of NLF treatment with fillers, patients complain about superolateral migration of the filler despite inferomedial filler injection. The movement of the expression muscles may lead to a shift of the injected filler to an area of lower resistance.

In the present case, a combination therapy of the polydioxanone (PDO) thread in the form of mesh tube and hyaluronic acid (HA) filler to restore the volume of the NLF and prevent filler migration was performed. The softening of the NLF without filler migration and a natural charming smile with labiomandibular fold improvement was observed concomitantly on combining the filler and mesh thread. We would like to present this case related to the tissue tightening and myo-modulating effects of mesh thread and HA filler.

\section{Case report}

\section{Patient selection}

A 67-year-old woman who had not undergone any facial procedures in the previous 5 years volunteered for the treatment protocol. In addition, each patient was given an opportunity to review the manuscript and consented to its publication.

\section{Procedure materials}

PDO thread in the form of mesh tube (Essorb; PineBM Co, Ltd, Daejeon, Korea) and HA filler (Polyderm) were used in the
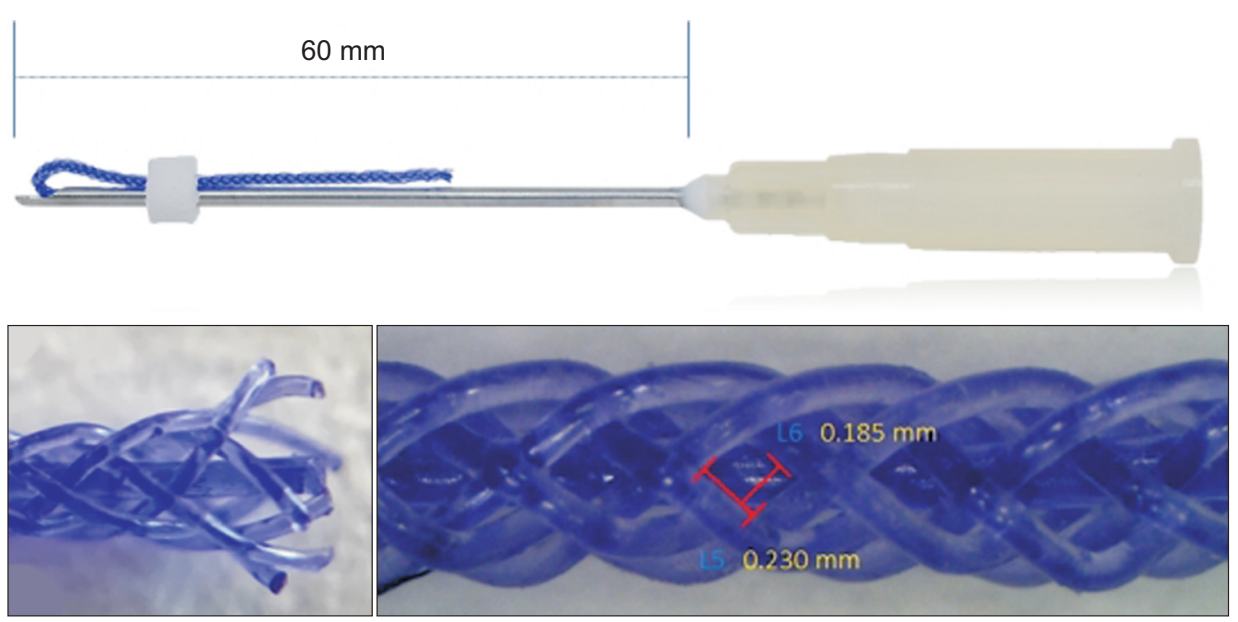

Fig. 2. Schematic figure of the polydioxanone thread device (Essorb). A core thread (4.0 USP) is surrounded by 8 peripheral threads (6.0 USP) with switch type and is equipped to the cannula (19 gauge; $60 \mathrm{~mm}$ in length). 
procedure (Fig. 2).

\section{Polydioxanone thread insertion protocol}

Local anesthesia using a $2 \%$ lidocaine solution with epinephrine $(1: 100,000)$ was administered at the entry site and modified Klein's tumescent solution was infiltrated into the NLF area.

NLF area was divided into three parts: lateral folded part (LFP), medial depressed part (MDP), and central crease part (CCP) (Fig. 3A). Five threads were inserted on each side, one each in the submuscular and subcutaneous fat layers of the
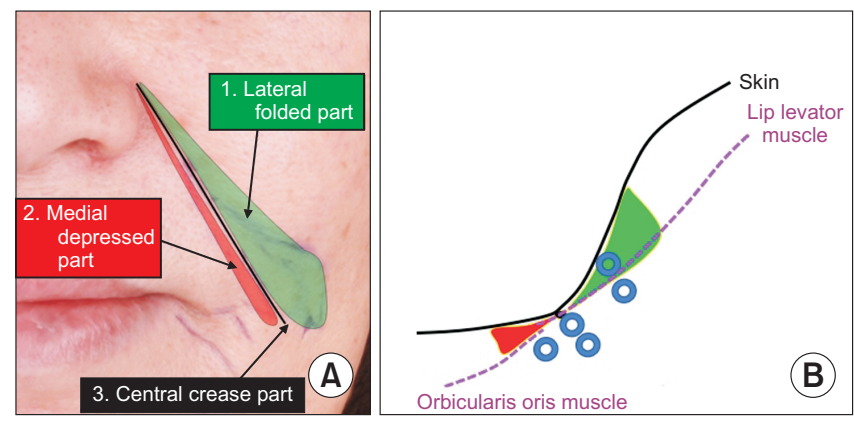

Fig. 3. (A) Facial design for mesh thread and filler injection for the nasolabial fold (NLF). Three parts of NLF; 1 ) green colored lateral folded part, 2) red colored medial depressed part, and 3) black line of central crease part. (B) Schematic showing different placement of the threads from the cross-sectional view of NLF. folded part, one in the subcutaneous fat layer of the depressed part, and one each in the submuscular and subcutaneous fat layers of the crease (Fig. 3B).

After inserting volume mesh threads, filler was sequentially injected into the LFP, MDP, and CCP. As with thread insertion, a total of $2.0 \mathrm{ml}$ was injected into one side in the order of the submuscular and subcutaneous fat layers. With regards to the LFP, thread and filler were injected to tighten and thicken the tissue so that it did not fold, and in the MDP region, it was aimed to compensate for the defect by increasing the volume and eliminate the linear line in the CCP region.

\section{Results}

Three months after PDO threads and HA filler procedure, several significant cosmetic improvements including softened NLFs without filler migration, restored volume and lifted midface with a natural and charming smile, and reduction of perioral wrinkles were observed. Fig. 4 presents non-smiling views. A preoperative photograph shows increased contraction of the lip elevator and risorius muscles in the NLFs and perioral wrinkles (Fig. 4A). Fig. 4B and C illustrate the immediate and one hour after the procedure images, respectively, showing the dramatic change in softening of NLFs and volume increase in the mid-cheek furrow. At the three-month follow up (Fig. 4D), an unfolded and flattened LFP, volume-augmented MDP, and
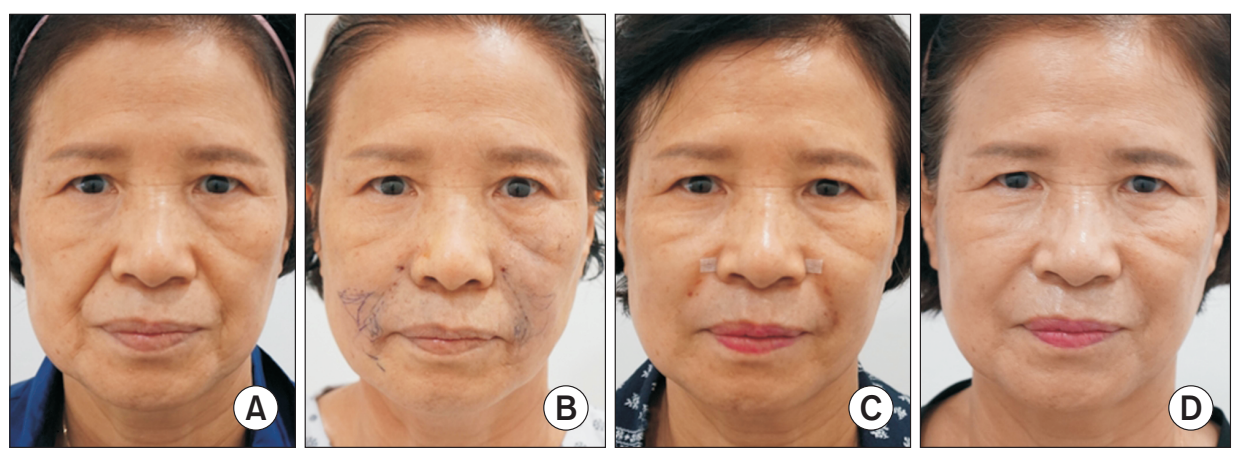

Fig. 4. Non-smiling views; combination effect of the thread and hyaluronic acid filler on the correction of deep nasolabial folds in a 67 -year-old woman. (A) Preoperative, (B) immediately after the procedure, (C) one hour after the procedure, and (D) three months after the procedure.
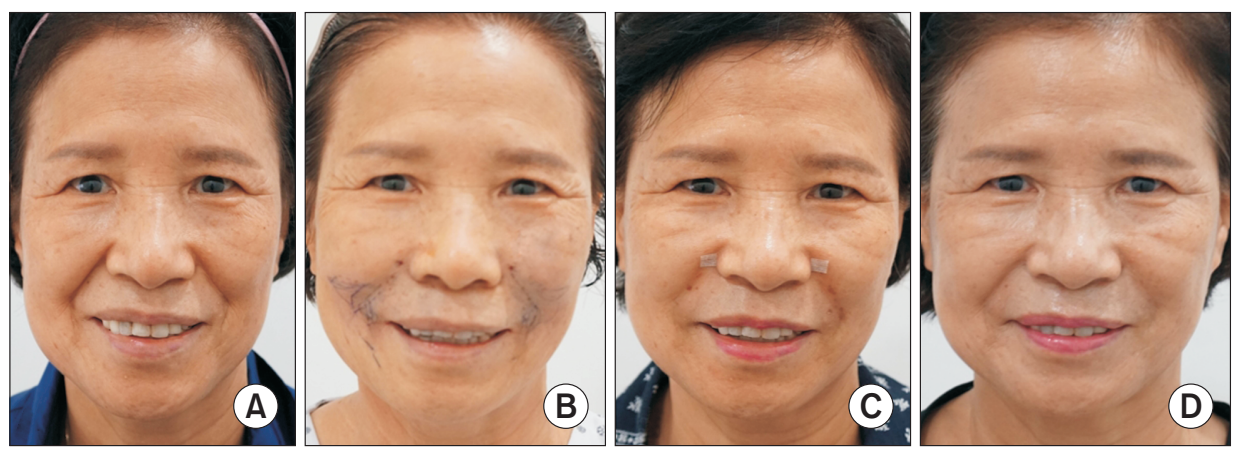

Fig. 5. Smiling views; combination effects of the thread and hyaluronic acid filler on the correction of deep nasolabial folds in a 67-year-old woman. (A) Preoperative, (B) immediately after the procedure, (C) one hour after the procedure, and (D) three months after the procedure. 
lifted midface with fewer wrinkles were observed.

Fig. 5 presents the smiling views reflecting the change in the smile-related muscles after the PDO thread and HA filler combination procedure. A preoperative photograph showed an increased basic tone of contraction of the lip elevator and risorius muscle in the NLFs and perioral wrinkles (Fig. 5A). In the smiling views immediately after and one hour after the procedure (Fig. 5B, C), the LFP of the NLF was unfolded, the volume recovery of the MDP of the fold was also successful, and her smile appeared more natural. In the image of three months later (Fig. $5 \mathrm{D})$, the most noticeable change was that the teeth exposure had decreased, giving a natural and charming smile with volumizing of the midface resulting in a youthful appearance.

\section{Discussion}

In elderly patients, the NLFs typically show superficial submalar fat and skin folding, medial depression with a central crease, and connection with the volume loss of the DMCF laterally and the perioral wrinkles inferiorly. The approach in the present case was to achieve local volume restoration of the NLF and prevent lateral migration of the injected filler. In addition to the volume expansion and filler movement prevention observed in another study [1], our results showed the lifted appearance of the mid- and lower-face with increased midface volume, softening of mid-cheek furrows, reduction of perioral wrinkles and labio-mandibular folds, and natural smiling appearance.

The lifting of the mid and lower face appeared to be due to both tightening by the PDO thread and volumizing by the filler. According to Hwang et al. [8], thread acts as a tissue scaffold, and activates intercellular interactions, collagen deposition, neovascularization, and mechanotransduction. Finally, it tightens and thickens the skin and subcutaneous fat. Volumized dermis and superficial and deep fat layers contributed to the unfolding of the LFP of the NLF and the augmentation of MDP and CCP of the fold, making NLF tighter and the firm and augmented NLF pushed the mid-cheek superolaterally. Finally, the midface was volumized and lifted.

According to de Maio [7], patients with certain structural deficiencies treated only with fillers had the alterations in muscle movement (myo-modulation). He emphasized the influence of muscles on the balance of the face and argued that controlling muscle movements with fillers compensates for structural defects, corrects abnormal muscle movements, and establishes a normal facial appearance.
In our study, the increased basic tone of contraction of the lip elevators, risorius, and orbicularis oris (OOr) muscles was seen in the NLFs and perioral wrinkles preoperatively, but a very comfortable look and fewer wrinkles around the mouth were seen postoperatively, which might have been caused by decreased muscle contraction. This was caused by muscular compensation against aging due to sagging of the midface followed by decreased firmness of the NLFs and volume loss of the midface. Fitzgerald et al. [2] argued that, as we age, muscle contraction and stiffness increase as a reflex to support soft tissue absorption and sag. It is expected that the threads and HA filler placed either over or under the contracted lip elevators and OOr muscle would be involved in the myo-modulation of these muscles, allowing the muscles to relax, alleviating the NLFs, and helping the natural smile. As these muscles relaxed, the strong contraction of the muscles when laughing was suppressed, so the NLF softened, and the smile became more natural.

To date, with respect to the procedure to correct NLFs, many doctors have focused on the injection technique (fanning, liner, cross-linked technique, etc.) necessary to restore the volume of the bulging area. However, although the present study included only one case, the results suggest the possibility of developing an academic and systematic treatment modality for NLFs in which the aging process, facial animation, and related soft tissue structures and functions are considered thoroughly.

More cases and long-term follow are necessary to demonstrate the efficiency and safety of this combination method.

\section{Conflicts of interest}

The authors have nothing to disclose.

\section{References}

1. Han SE, Go JY, Pyon JK, Oh KS. A prospective evaluation of outcomes for midface rejuvenation with mesh suspension thread: “REEBORN lift”. J Cosmet Dermatol 2016;15:254-9.

2. Fitzgerald R, Graivier MH, Kane M, Lorenc ZP, Vleggaar D, Werschler WP, et al. Update on facial aging. Aesthet Surg J 2010;30 Suppl:11S-24S.

3. Hur MS, O J, Yang HM, Kwon HJ, Lee S, Lim HS, et al. Heights and spatial relationships of the facial muscles acting on the nasolabial fold by dissection and three-dimensional microcomputed tomography. PLoS One 2020;15:e0237043.

4. Beer GM, Manestar M, Mihic-Probst D. The causes of the nasolabial crease: a histomorphological study. Clin Anat 
2013;26:196-203.

5. Chung C, Lee JH. A single-center, randomized, double-blind clinical trial to compare the efficacy and safety of a new monophasic hyaluronic acid filler and biphasic filler in correcting nasolabial fold. Aesthetic Plast Surg 2021;45:2902-8.

6. Raspaldo H, Baspeyras M, Bellity P, Dallara JM, Gassia V, Niforos FR, et al.; Consensus Group. Upper- and mid-face antiaging treatment and prevention using onabotulinumtoxin $\mathrm{A}$ : the 2010 multidisciplinary French consensus--part 1. J Cosmet Dermatol 2011;10:36-50.

7. de Maio M. Myomodulation with injectable fillers: an innovative approach to addressing facial muscle movement. Aesthetic Plast Surg 2018;42:798-814.

8. Hwang UK, Kwon YH, Cho YJ, Kang KJ. Improvement of aged lower eyelid by insertion of polydioxanone threads. J Cosmet Med 2017;1:57-9. 